\title{
A sharpened and generalized version of Aczél-Vasić-Pečarić inequality and its application
}

Jingfeng Tian*

\section{${ }^{\text {*Correspondence: }}$}

tianjfhxm_ncepu@163.com

College of Science and Technology,

North China Electric Power

University, Baoding, Hebei Province

071051, P.R. China

\section{Abstract \\ In this paper, we present a sharpened and generalized version of Aczél-Vasić-Pečarić inequality. As an application, an integral type of Aczél-Vasić-Pečarić inequality is obtained. \\ MSC: Primary 26D15; secondary 26D10 \\ Keywords: Aczél's inequality; Aczél-Vasić-Pečarić inequality; Hölder's inequality}

\section{Introduction}

In 1956, Aczél [1] established the following inequality, which is of wide application in the theory of functional equations in non-Euclidean geometry.

Theorem A If $a_{i}, b_{i}(i=1,2, \ldots, n)$ are positive numbers such that $a_{1}^{2}-\sum_{i=2}^{n} a_{i}^{2}>0$ or $b_{1}^{2}-\sum_{i=2}^{n} b_{i}^{2}>0$, then

$$
\left(a_{1}^{2}-\sum_{i=2}^{n} a_{i}^{2}\right)\left(b_{1}^{2}-\sum_{i=2}^{n} b_{i}^{2}\right) \leq\left(a_{1} b_{1}-\sum_{i=2}^{n} a_{i} b_{i}\right)^{2} .
$$

Later, in 1959, Popoviciu [2] gave a generalization of the above inequality.

Theorem B Let $p>1, q>1, \frac{1}{p}+\frac{1}{q}=1$, and let $a_{i}, b_{i}(i=1,2, \ldots, n)$ be positive numbers such that $a_{1}^{p}-\sum_{i=2}^{n} a_{i}^{p}>0$ and $b_{1}^{q}-\sum_{i=2}^{n} b_{i}^{q}>0$. Then

$$
\left(a_{1}^{p}-\sum_{i=2}^{n} a_{i}^{p}\right)^{\frac{1}{p}}\left(b_{1}^{q}-\sum_{i=2}^{n} b_{i}^{q}\right)^{\frac{1}{q}} \leq a_{1} b_{1}-\sum_{i=2}^{n} a_{i} b_{i} .
$$

In 1982, Vasić and Pečarić [3] presented the following reversed version of inequality (2).

Theorem C Let $p<1(p \neq 0), \frac{1}{p}+\frac{1}{q}=1$, and let $a_{i}, b_{i}(i=1,2, \ldots, n)$ be positive numbers such that $a_{1}^{p}-\sum_{i=2}^{n} a_{i}^{p}>0$ and $b_{1}^{q}-\sum_{i=2}^{n} b_{i}^{q}>0$. Then

$$
\left(a_{1}^{p}-\sum_{i=2}^{n} a_{i}^{p}\right)^{\frac{1}{p}}\left(b_{1}^{q}-\sum_{i=2}^{n} b_{i}^{q}\right)^{\frac{1}{q}} \geq a_{1} b_{1}-\sum_{i=2}^{n} a_{i} b_{i} .
$$

○2013 Tian; licensee Springer. This is an Open Access article distributed under the terms of the Creative Commons Attribution License (http://creativecommons.org/licenses/by/2.0), which permits unrestricted use, distribution, and reproduction in any medium, provided the original work is properly cited. 
Recently inequalities (2) and (3) were generalized and refined in many different ways; see, for example, [4-10] and [11]. In [12], Wu established an interesting generalization of Aczél-Popoviciu inequality (2) as follows.

Theorem D Let $p, q>0, a_{i}, b_{i}>0(i=1,2, \ldots, n)$, let $k(1 \leq k<n)$ be a positive integer such that $\sum_{i=1}^{k} a_{i}^{p}-\sum_{i=k+1}^{n} a_{i}^{p}>0$ and $\sum_{i=1}^{k} b_{i}^{q}-\sum_{i=k+1}^{n} b_{i}^{q}>0$. Then

$$
\begin{aligned}
& \left(\sum_{i=1}^{k} a_{i}^{p}-\sum_{i=k+1}^{n} a_{i}^{p}\right)^{\frac{1}{p}}\left(\sum_{i=1}^{k} b_{i}^{q}-\sum_{i=k+1}^{n} b_{i}^{q}\right)^{\frac{1}{q}} \\
& \leq 2^{\max \left\{1-\frac{1}{p}-\frac{1}{q}, 0\right\}}\left(\sum_{i=1}^{k} a_{i}^{p}\right)^{\frac{1}{p}}\left(\sum_{i=1}^{k} b_{i}^{q}\right)^{\frac{1}{q}}-\left(\sum_{i=k+1}^{n} a_{i}^{p}\right)^{\frac{1}{p}}\left(\sum_{i=k+1}^{n} b_{i}^{q}\right)^{\frac{1}{q}} \\
& \quad-\frac{2^{\max \left\{1-\frac{1}{p}-\frac{1}{q}, 0\right\}}}{\max \{p, q, 1\}}\left(\sum_{i=1}^{k} a_{i}^{p}\right)^{\frac{1}{p}}\left(\sum_{i=1}^{k} b_{i}^{q}\right)^{\frac{1}{q}}\left(\frac{\sum_{i=k+1}^{n} a_{i}^{p}}{\sum_{i=1}^{k} a_{i}^{p}}-\frac{\sum_{i=k+1}^{n} b_{i}^{q}}{\sum_{i=1}^{k} b_{i}^{q}}\right)^{2},
\end{aligned}
$$

and equality holds if and only if

$$
\frac{\sum_{i=1}^{k} a_{i}^{p}}{\sum_{i=k+1}^{n} a_{i}^{p}}=\frac{\sum_{i=1}^{k} b_{i}^{q}}{\sum_{i=k+1}^{n} b_{i}^{q}}=2
$$

for $\frac{1}{p}+\frac{1}{q}<1$ or

$$
\frac{\sum_{i=1}^{k} a_{i}^{p}}{\sum_{i=k+1}^{n} a_{i}^{p}}=\frac{\sum_{i=1}^{k} b_{i}^{q}}{\sum_{i=k+1}^{n} b_{i}^{q}}
$$

for $\frac{1}{p}+\frac{1}{q}=1$.

The main purpose of this work is to give a sharpened and generalized version of AczélVasić-Pečarić inequality (3). Moreover, a new Aczél-Vasić-Pečarić type integral inequality is established.

\section{A sharpened and generalized version of Aczél-Vasić-Pečarić inequality}

We begin this section with some lemmas, which will be used in the sequel.

Lemma 2.1 [13] If $x>-1, \alpha \geq 1$ or $\alpha<0$, then

$$
(1+x)^{\alpha} \geq 1+\alpha x .
$$

The inequality is reversed for $0<\alpha<1$. The sign of equality holds if and only if $x=0$ or $\alpha=1$.

Lemma 2.2 [14] Let $a_{r j}>0(r=1,2, \ldots, n, j=1,2, \ldots, m)$, let $\lambda_{1} \neq 0, \lambda_{j}<0(j=2,3, \ldots, m)$, and let $\tau=\max \left\{\sum_{j=1}^{m} \frac{1}{\lambda_{j}}, 1\right\}$. Then

$$
\sum_{r=1}^{n} \prod_{j=1}^{m} a_{r j} \geq n^{1-\tau} \prod_{j=1}^{m}\left(\sum_{r=1}^{n} a_{r j}^{\lambda_{j}}\right)^{\frac{1}{\lambda_{j}}} .
$$


The sign of equality holds if and only if the $m$ sets $\left(a_{r 1}\right),\left(a_{r 2}\right), \ldots,\left(a_{r m}\right)$ are proportional for $\sum_{j=1}^{m} \frac{1}{\lambda_{j}} \leq 1$, or $a_{1 j}=a_{2 j}=\cdots=a_{n j}, j=1,2, \ldots, m$, for $\sum_{j=1}^{m} \frac{1}{\lambda_{j}}>1$.

Lemma 2.3 Let $x>1, y>1$, and let $p<0, q<0$. Then

$$
x y+\left(1-x^{p}\right)^{\frac{1}{p}}\left(1-y^{q}\right)^{\frac{1}{q}} \geq 2^{\min \left\{1-\frac{1}{p}-\frac{1}{q}, 0\right\}}\left(1-\min \left\{p^{-1}, q^{-1}\right\}\left(x^{p}-y^{q}\right)^{2}\right),
$$

and equality holds if and only if $x^{p}=y^{q}=\frac{1}{2}$.

Proof Case (I). When $p<q<0$, it implies that $\frac{1}{q}<0, \frac{1}{p}-\frac{1}{q}>0$. By applying Lemma 2.2 and Lemma 2.1, we have

$$
\begin{aligned}
x y & +\left(1-x^{p}\right)^{\frac{1}{p}}\left(1-y^{q}\right)^{\frac{1}{q}} \\
& =\left(x^{p}\right)^{\frac{1}{q}}\left(y^{q}\right)^{\frac{1}{q}}\left(x^{p}\right)^{\frac{1}{p}-\frac{1}{q}}+\left(1-y^{q}\right)^{\frac{1}{q}}\left(1-x^{p}\right)^{\frac{1}{q}}\left(1-x^{p}\right)^{\frac{1}{p}-\frac{1}{q}} \\
& \geq 2^{\min \left\{1-\frac{1}{p}-\frac{1}{q}, 0\right\}}\left(x^{p}+\left(1-y^{q}\right)\right)^{\frac{1}{q}}\left(y^{q}+\left(1-x^{p}\right)\right)^{\frac{1}{q}}\left(x^{p}+\left(1-x^{p}\right)\right)^{\frac{1}{p}-\frac{1}{q}} \\
& =2^{\min \left\{1-\frac{1}{p}-\frac{1}{q}, 0\right\}}\left(1-\left(x^{p}-y^{q}\right)^{2}\right)^{\frac{1}{q}} \\
& \geq 2^{\min \left\{1-\frac{1}{p}-\frac{1}{q}, 0\right\}}\left(1-q^{-1}\left(x^{p}-y^{q}\right)^{2} .\right.
\end{aligned}
$$

Case (II). When $q<p<0$, it implies that $\frac{1}{p}<0, \frac{1}{q}-\frac{1}{p}>0$. By using Lemma 2.2 and Lemma 2.1, we obtain

$$
\begin{aligned}
x y & +\left(1-x^{p}\right)^{\frac{1}{p}}\left(1-y^{q}\right)^{\frac{1}{q}} \\
& =\left(y^{q}\right)^{\frac{1}{p}}\left(x^{p}\right)^{\frac{1}{p}}\left(y^{q}\right)^{\frac{1}{q}-\frac{1}{p}}+\left(1-x^{p}\right)^{\frac{1}{p}}\left(1-y^{q}\right)^{\frac{1}{p}}\left(1-y^{q}\right)^{\frac{1}{q}-\frac{1}{p}} \\
& \geq 2^{\min \left\{1-\frac{1}{p}-\frac{1}{q}, 0\right\}}\left(y^{q}+\left(1-x^{p}\right)\right)^{\frac{1}{p}}\left(x^{p}+\left(1-y^{q}\right)\right)^{\frac{1}{p}}\left(y^{q}+\left(1-y^{q}\right)\right)^{\frac{1}{q}-\frac{1}{p}} \\
& =2^{\min \left\{1-\frac{1}{p}-\frac{1}{q}, 0\right\}}\left(1-\left(x^{p}-y^{q}\right)^{2}\right)^{\frac{1}{p}} \\
& \geq 2^{\min \left\{1-\frac{1}{p}-\frac{1}{q}, 0\right\}}\left(1-p^{-1}\left(x^{p}-y^{q}\right)^{2} .\right.
\end{aligned}
$$

Case (III). When $p=q, p<0, q<0$. From Lemma 2.2 and Lemma 2.1 we have

$$
\begin{aligned}
x y & +\left(1-x^{p}\right)^{\frac{1}{p}}\left(1-y^{q}\right)^{\frac{1}{q}} \\
& =\left(y^{p}\right)^{\frac{1}{p}}\left(x^{p}\right)^{\frac{1}{p}}+\left(1-x^{p}\right)^{\frac{1}{p}}\left(1-y^{p}\right)^{\frac{1}{p}} \\
& \geq 2^{\min \left\{1-\frac{2}{p}, 0\right\}}\left(y^{p}+\left(1-x^{p}\right)\right)^{\frac{1}{p}}\left(x^{p}+\left(1-y^{p}\right)\right)^{\frac{1}{p}} \\
& =2^{\min \left\{1-\frac{2}{p}, 0\right\}}\left(1-\left(x^{p}-y^{p}\right)^{2}\right)^{\frac{1}{p}} \\
& =2^{\min \left\{1-\frac{1}{p}-\frac{1}{q}, 0\right\}}\left(1-\left(x^{p}-y^{q}\right)^{2}\right)^{\frac{1}{p}} \\
& \geq 2^{\min \left\{1-\frac{1}{p}-\frac{1}{q}, 0\right\}}\left(1-p^{-1}\left(x^{p}-y^{q}\right)^{2}\right) .
\end{aligned}
$$

Combining inequalities (8)-(10) yields inequality (7). The condition of equality in (7) follows immediately from Lemma 2.2 and Lemma 2.1. The proof of Lemma 2.3 is completed. 
Lemma 2.4 Let $0<x<1, y>1$, and let $p>0, q<0$. Then

$$
x y+\left(1-x^{p}\right)^{\frac{1}{p}}\left(1-y^{q}\right)^{\frac{1}{q}} \geq 2^{\min \left\{1-\frac{1}{p}-\frac{1}{q}, 0\right\}}\left(1-\min \left\{p^{-1}, q^{-1}\right\}\left(x^{p}-y^{q}\right)^{2}\right),
$$

and equality holds if and only if $x^{p}=y^{q}=\frac{1}{2}$ for $\frac{1}{p}+\frac{1}{q}<1$, or $x^{p}=y^{q}$ for $\frac{1}{p}+\frac{1}{q}=1$.

Proof By using Lemma 2.2 and Lemma 2.1, we have

$$
\begin{aligned}
x y & +\left(1-x^{p}\right)^{\frac{1}{p}}\left(1-y^{q}\right)^{\frac{1}{q}} \\
& =\left(x^{p}\right)^{\frac{1}{q}}\left(y^{q}\right)^{\frac{1}{q}}\left(x^{p}\right)^{\frac{1}{p}-\frac{1}{q}}+\left(1-y^{q}\right)^{\frac{1}{q}}\left(1-x^{p}\right)^{\frac{1}{q}}\left(1-x^{p}\right)^{\frac{1}{p}-\frac{1}{q}} \\
& \geq 2^{\min \left\{1-\frac{1}{p}-\frac{1}{q}, 0\right\}}\left(x^{p}+\left(1-y^{q}\right)\right)^{\frac{1}{q}}\left(y^{q}+\left(1-x^{p}\right)\right)^{\frac{1}{q}}\left(x^{p}+\left(1-x^{p}\right)\right)^{\frac{1}{p}-\frac{1}{q}} \\
& =2^{\min \left\{1-\frac{1}{p}-\frac{1}{q}, 0\right\}}\left(1-\left(x^{p}-y^{q}\right)^{2}\right)^{\frac{1}{q}} \\
& \geq 2^{\min \left\{1-\frac{1}{p}-\frac{1}{q}, 0\right\}}\left(1-q^{-1}\left(x^{p}-y^{q}\right)^{2}\right) \\
& =2^{\min \left\{1-\frac{1}{p}-\frac{1}{q}, 0\right\}}\left(1-\min \left\{p^{-1}, q^{-1}\right\}\left(x^{p}-y^{q}\right)^{2}\right) .
\end{aligned}
$$

In addition, the condition of equality for inequality (11) can easily be obtained by Lemma 2.1 and Lemma 2.2. The proof of Lemma 2.4 is completed.

Theorem 2.5 Let $a_{i}>0, b_{i}>0(i=1,2, \ldots, n)$, let $p \neq 0, q<0$, and let $k(1 \leq k<n)$ be $a$ positive integer such that $\sum_{i=1}^{k} a_{i}^{p}-\sum_{i=k+1}^{n} a_{i}^{p}>0$ and $\sum_{i=1}^{k} b_{i}^{q}-\sum_{i=k+1}^{n} b_{i}^{q}>0$. Then

$$
\begin{aligned}
& \left(\sum_{i=1}^{k} a_{i}^{p}-\sum_{i=k+1}^{n} a_{i}^{p}\right)^{\frac{1}{p}}\left(\sum_{i=1}^{k} b_{i}^{q}-\sum_{i=k+1}^{n} b_{i}^{q}\right)^{\frac{1}{q}} \\
& \geq 2^{\min \left\{1-\frac{1}{p}-\frac{1}{q}, 0\right\}}\left(\sum_{i=1}^{k} a_{i}^{p}\right)^{\frac{1}{p}}\left(\sum_{i=1}^{k} b_{i}^{q}\right)^{\frac{1}{q}}-\left(\frac{1}{n-k}\right)^{\min \left\{1-\frac{1}{p}-\frac{1}{q}, 0\right\}}\left(\sum_{i=k+1}^{n} a_{i} b_{i}\right) \\
& -2^{\min \left\{1-\frac{1}{p}-\frac{1}{q}, 0\right\}} \min \left\{p^{-1}, q^{-1}\right\}\left(\sum_{i=1}^{k} a_{i}^{p}\right)^{\frac{1}{p}}\left(\sum_{i=1}^{k} b_{i}^{q}\right)^{\frac{1}{q}} \\
& \quad \times\left(\frac{\sum_{i=k+1}^{n} a_{i}^{p}}{\sum_{i=1}^{k} a_{i}^{p}}-\frac{\sum_{i=k+1}^{n} b_{i}^{q}}{\sum_{i=1}^{k} b_{i}^{q}}\right)^{2}
\end{aligned}
$$

and equality holds if and only if $(2 n-2 k)^{-1} \sum_{i=1}^{k} a_{i}^{p}=a_{k+1}^{p}=a_{k+2}^{p}=\cdots=a_{n}^{p}$ and (2n$2 k)^{-1} \sum_{i=1}^{k} b_{i}^{q}=b_{k+1}^{q}=b_{k+2}^{q}=\cdots=b_{n}^{q}$ for $\frac{1}{p}+\frac{1}{q}<1$, or $\frac{\sum_{i=1}^{k} a_{i}^{p}}{\sum_{i=1}^{k} b_{i}^{q}}=\frac{a_{k+1}^{p}}{b_{k+1}^{q}}=\frac{a_{k+2}^{p}}{b_{k+2}^{q}}=\cdots=\frac{a_{n}^{p}}{b_{n}^{q}}$ for $\frac{1}{p}+\frac{1}{q}=1$.

Proof Case (I). When $p>0, q<0$. From the hypotheses of Theorem 2.5, we find that

$$
\begin{aligned}
& 0<\left(\frac{\sum_{i=1}^{k} a_{i}^{p}-\sum_{i=k+1}^{n} a_{i}^{p}}{\sum_{i=1}^{k} a_{i}^{p}}\right)^{\frac{1}{p}}<1, \\
& \left(\frac{\sum_{i=1}^{k} b_{i}^{q}-\sum_{i=k+1}^{n} b_{i}^{q}}{\sum_{i=1}^{k} b_{i}^{q}}\right)^{\frac{1}{q}}>1 .
\end{aligned}
$$


Thus, by using Lemma 2.4 with a substitution

$$
x=\left(\frac{\sum_{i=1}^{k} a_{i}^{p}-\sum_{i=k+1}^{n} a_{i}^{p}}{\sum_{i=1}^{k} a_{i}^{p}}\right)^{\frac{1}{p}}, \quad y=\left(\frac{\sum_{i=1}^{k} b_{i}^{q}-\sum_{i=k+1}^{n} b_{i}^{q}}{\sum_{i=1}^{k} b_{i}^{q}}\right)^{\frac{1}{q}}
$$

in (11), we have

$$
\begin{aligned}
& \left(\frac{\sum_{i=1}^{k} a_{i}^{p}-\sum_{i=k+1}^{n} a_{i}^{p}}{\sum_{i=1}^{k} a_{i}^{p}}\right)^{\frac{1}{p}}\left(\frac{\sum_{i=1}^{k} b_{i}^{q}-\sum_{i=k+1}^{n} b_{i}^{q}}{\sum_{i=1}^{k} b_{i}^{q}}\right)^{\frac{1}{q}}+\left(\frac{\sum_{i=k+1}^{n} a_{i}^{p}}{\sum_{i=1}^{k} a_{i}^{p}}\right)^{\frac{1}{p}}\left(\frac{\sum_{i=k+1}^{n} b_{i}^{q}}{\sum_{i=1}^{k} b_{i}^{q}}\right)^{\frac{1}{q}} \\
& \geq 2^{\min \left\{1-\frac{1}{p}-\frac{1}{q}, 0\right\}} \\
& \quad \times\left[1-\min \left\{p^{-1}, q^{-1}\right\}\left(\frac{\sum_{i=1}^{k} a_{i}^{p}-\sum_{i=k+1}^{n} a_{i}^{p}}{\sum_{i=1}^{k} a_{i}^{p}}-\frac{\sum_{i=1}^{k} b_{i}^{q}-\sum_{i=k+1}^{n} b_{i}^{q}}{\sum_{i=1}^{k} b_{i}^{q}}\right)^{2}\right]
\end{aligned}
$$

which implies

$$
\begin{gathered}
\left(\sum_{i=1}^{k} a_{i}^{p}-\sum_{i=k+1}^{n} a_{i}^{p}\right)^{\frac{1}{p}}\left(\sum_{i=1}^{k} b_{i}^{q}-\sum_{i=k+1}^{n} b_{i}^{q}\right)^{\frac{1}{q}}+\left(\sum_{i=k+1}^{n} a_{i}^{p}\right)^{\frac{1}{p}}\left(\sum_{i=k+1}^{n} b_{i}^{q}\right)^{\frac{1}{q}} \\
\geq 2^{\min \left\{1-\frac{1}{p}-\frac{1}{q}, 0\right\}}\left(\sum_{i=1}^{k} a_{i}^{p}\right)^{\frac{1}{p}}\left(\sum_{i=1}^{k} b_{i}^{q}\right)^{\frac{1}{q}} \\
\times\left[1-\min \left\{p^{-1}, q^{-1}\right\}\left(\frac{\sum_{i=k+1}^{n} a_{i}^{p}}{\sum_{i=1}^{k} a_{i}^{p}}-\frac{\sum_{i=k+1}^{n} b_{i}^{q}}{\sum_{i=1}^{k} b_{i}^{q}}\right)^{2}\right] .
\end{gathered}
$$

Hence, we obtain

$$
\begin{aligned}
& \left(\sum_{i=1}^{k} a_{i}^{p}-\sum_{i=k+1}^{n} a_{i}^{p}\right)^{\frac{1}{p}}\left(\sum_{i=1}^{k} b_{i}^{q}-\sum_{i=k+1}^{n} b_{i}^{q}\right)^{\frac{1}{q}} \\
& \geq 2^{\min \left\{1-\frac{1}{p}-\frac{1}{q}, 0\right\}}\left(\sum_{i=1}^{k} a_{i}^{p}\right)^{\frac{1}{p}}\left(\sum_{i=1}^{k} b_{i}^{q}\right)^{\frac{1}{q}}-\left(\sum_{i=k+1}^{n} a_{i}^{p}\right)^{\frac{1}{p}}\left(\sum_{i=k+1}^{n} b_{i}^{q}\right)^{\frac{1}{q}} \\
& \quad-2^{\min \left\{1-\frac{1}{p}-\frac{1}{q}, 0\right\}} \min \left\{p^{-1}, q^{-1}\right\} \\
& \quad \times\left(\sum_{i=1}^{k} a_{i}^{p}\right)^{\frac{1}{p}}\left(\sum_{i=1}^{k} b_{i}^{q}\right)^{\frac{1}{q}}\left(\frac{\sum_{i=k+1}^{n} a_{i}^{p}}{\sum_{i=1}^{k} a_{i}^{p}}-\frac{\sum_{i=k+1}^{n} b_{i}^{q}}{\sum_{i=1}^{k} b_{i}^{q}}\right)^{2}
\end{aligned}
$$

where the equality holds if and only if $\frac{\sum_{i=k+1}^{n} a_{i}^{p}}{\sum_{i=1}^{k} a_{i}^{p}}=\frac{\sum_{i=k+1}^{n} b_{i}^{q}}{\sum_{i=1}^{k} b_{i}^{q}}=\frac{1}{2}$ for $\frac{1}{p}+\frac{1}{q}<1$, or $\frac{\sum_{i=k+1}^{n} a_{i}^{p}}{\sum_{i=1}^{k} a_{i}^{p}}=$ $\frac{\sum_{i=k+1}^{n} b_{i}^{q}}{\sum_{i=1}^{k} b_{i}^{q}}$ for $\frac{1}{p}+\frac{1}{q}=1$.

On the other hand, by using Lemma 2.2, we obtain

$$
\left(\sum_{i=k+1}^{n} a_{i}^{p}\right)^{\frac{1}{p}}\left(\sum_{i=k+1}^{n} b_{i}^{q}\right)^{\frac{1}{q}} \leq\left(\frac{1}{n-k}\right)^{\min \left\{1-\frac{1}{p}-\frac{1}{q}, 0\right\}}\left(\sum_{i=k+1}^{n} a_{i} b_{i}\right),
$$

where the equality holds if and only if $a_{k+1}=a_{k+2}=\cdots=a_{n}$ and $b_{k+1}=b_{k+2}=\cdots=b_{n}$ for $\frac{1}{p}+\frac{1}{q}<1$, or $\frac{a_{k+1}^{p}}{b_{k+1}^{q}}=\frac{a_{k+2}^{p}}{b_{k+2}^{q}}=\cdots=\frac{a_{n}^{p}}{b_{n}^{q}}$ for $\frac{1}{p}+\frac{1}{q}=1$. 
Combining the above two inequalities gives the desired result.

Case (II). When $p<0, q<0$. By the same method as in the above case (I) and using Lemma 2.3 and Lemma 2.2, we get that inequality (13) is also valid. The proof of Theorem 2.5 is completed.

Remark 2.6 If we set $k=1, \frac{1}{p}+\frac{1}{q}=1$, and $\frac{\sum_{i=2}^{n} a_{i}^{p}}{a_{1}^{p}}=\frac{\sum_{i=2}^{n} b_{i}^{q}}{b_{1}^{q}}$ in Theorem 2.5 , then inequality (13) reduces to inequality (3).

If we set $k=1$, then from Theorem 2.5 we obtain the following sharpened and generalized version of Aczél-Vasić-Pečarić inequality (3).

Corollary 2.7 Let $p \neq 0, q<0$, and let $a_{i}>0, b_{i}>0, a_{1}^{p}-\sum_{i=2}^{n} a_{i}^{p}>0, b_{1}^{q}-\sum_{i=2}^{n} b_{i}^{q}>0$ $(i=1,2, \ldots, n)$. Then

$$
\begin{aligned}
& \left(a_{1}^{p}-\sum_{i=2}^{n} a_{i}^{p}\right)^{\frac{1}{p}}\left(b_{1}^{q}-\sum_{i=2}^{n} b_{i}^{q}\right)^{\frac{1}{q}} \\
& \geq 2^{\min \left\{1-\frac{1}{p}-\frac{1}{q}, 0\right\}} a_{1} b_{1}-\left(\frac{1}{n-1}\right)^{\min \left\{1-\frac{1}{p}-\frac{1}{q}, 0\right\}}\left(\sum_{i=2}^{n} a_{i} b_{i}\right) \\
& \quad-2^{\min \left\{1-\frac{1}{p}-\frac{1}{q}, 0\right\}} \min \left\{p^{-1}, q^{-1}\right\} a_{1} b_{1}\left[\sum_{i=2}^{n}\left(\frac{a_{i}^{p}}{a_{1}^{p}}-\frac{b_{i}^{q}}{b_{1}^{q}}\right)\right]^{2},
\end{aligned}
$$

and equality holds if and only if $(2 n-2)^{-\frac{1}{p}} a_{1}=a_{2}=\cdots=a_{n}$ and $(2 n-2)^{-\frac{1}{p}} b_{1}=b_{2}=\cdots=b_{n}$ for $\frac{1}{p}+\frac{1}{q}<1$, or $\frac{a_{1}^{p}}{b_{1}^{q}}=\frac{a_{2}^{p}}{b_{2}^{q}}=\cdots=\frac{a_{n}^{p}}{b_{n}^{q}}$ for $\frac{1}{p}+\frac{1}{q}=1$.

In particular, if we set $\frac{1}{p}+\frac{1}{q} \leq 1$, then from Corollary 2.7 we get the sharpened version of Aczél-Vasić-Pečarić inequality (3) as follows.

Corollary 2.8 Let $p>0, q<0, \frac{1}{p}+\frac{1}{q} \leq 1$, and let $a_{i}>0, b_{i}>0, a_{1}^{p}-\sum_{i=2}^{n} a_{i}^{p}>0, b_{1}^{q}-$ $\sum_{i=2}^{n} b_{i}^{q}>0(i=1,2, \ldots, n)$. Then

$$
\begin{aligned}
& \left(a_{1}^{p}-\sum_{i=2}^{n} a_{i}^{p}\right)^{\frac{1}{p}}\left(b_{1}^{q}-\sum_{i=2}^{n} b_{i}^{q}\right)^{\frac{1}{q}} \\
& \geq a_{1} b_{1}-\left(\sum_{i=2}^{n} a_{i} b_{i}\right)-\frac{a_{1} b_{1}}{q}\left[\sum_{i=2}^{n}\left(\frac{a_{i}^{p}}{a_{1}^{p}}-\frac{b_{i}^{q}}{b_{1}^{q}}\right)\right]^{2},
\end{aligned}
$$

and equality holds if and only if $\frac{a_{2}^{p}}{b_{2}^{q}}=\frac{a_{3}^{p}}{b_{3}^{q}}=\cdots=\frac{a_{n}^{p}}{b_{n}^{q}}$ and $\frac{1}{p}+\frac{1}{q}=1$.

\section{Application}

As application of the above results, we establish here an integral type of Aczél-VasićPečarić inequality.

Theorem 3.1 Let $p>0, q<0, \frac{1}{p}+\frac{1}{q}=1$, let $A>0, B>0$, and let $f(x), g(x)$ be positive Riemann integrable functions on $[a, b]$ such that $A^{p}-\int_{a}^{b} f^{p}(x) \mathrm{d} x>0$ and $B^{q}-\int_{a}^{b} g^{q}(x) \mathrm{d} x>0$. 
Then

$$
\begin{aligned}
& \left(A^{p}-\int_{a}^{b} f^{p}(x) \mathrm{d} x\right)^{\frac{1}{p}}\left(B^{q}-\int_{a}^{b} g^{q}(x) \mathrm{d} x\right)^{\frac{1}{q}} \\
& \geq A B-\int_{a}^{b} f(x) g(x) \mathrm{d} x-\frac{A B}{q}\left[\int_{a}^{b}\left(\frac{f^{p}(x)}{A^{p}}-\frac{g^{q}(x)}{B^{q}}\right) \mathrm{d} x\right]^{2} .
\end{aligned}
$$

Proof For any positive integer $n$, we choose an equidistant partition of $[a, b]$ as

$$
\begin{aligned}
& a<a+\frac{b-a}{n}<\cdots<a+\frac{b-a}{n} k<\cdots<a+\frac{b-a}{n}(n-1)<b, \\
& x_{i}=a+\frac{b-a}{n} i, \quad i=0,1, \ldots, n, \quad \Delta x_{k}=\frac{b-a}{n}, \quad k=1,2, \ldots, n .
\end{aligned}
$$

Since

$$
A^{p}-\int_{a}^{b} f^{p}(x) \mathrm{d} x>0, \quad B^{q}-\int_{a}^{b} g^{q}(x) \mathrm{d} x>0,
$$

we have

$$
A^{p}-\lim _{n \rightarrow \infty} \sum_{k=1}^{n} f^{p}\left(a+\frac{k(b-a)}{n}\right) \frac{b-a}{n}>0,
$$

and

$$
B^{q}-\lim _{n \rightarrow \infty} \sum_{k=1}^{n} g^{q}\left(a+\frac{k(b-a)}{n}\right) \frac{b-a}{n}>0 .
$$

Hence, there exists a positive integer $N$ such that

$$
A^{p}-\sum_{k=1}^{n} f^{p}\left(a+\frac{k(b-a)}{n}\right) \frac{b-a}{n}>0,
$$

and

$$
B^{q}-\sum_{k=1}^{n} g^{q}\left(a+\frac{k(b-a)}{n}\right) \frac{b-a}{n}>0 \quad \text { for all } n>N .
$$

By using Corollary 2.8, we obtain that for any $n>N$, the following inequality holds:

$$
\begin{aligned}
& {\left[A^{p}-\sum_{k=1}^{n} f^{p}\left(a+\frac{k(b-a)}{n}\right) \frac{b-a}{n}\right]^{\frac{1}{p}}\left[B^{q}-\sum_{k=1}^{n} g^{q}\left(a+\frac{k(b-a)}{n}\right) \frac{b-a}{n}\right]^{\frac{1}{q}}} \\
& \geq A B-\sum_{k=1}^{n} f^{p}\left(a+\frac{k(b-a)}{n}\right) g^{q}\left(a+\frac{k(b-a)}{n}\right)\left(\frac{b-a}{n}\right)^{\frac{1}{p}+\frac{1}{q}} \\
& \quad-\frac{A B}{q}\left\{\sum_{k=1}^{n}\left[\frac{1}{A^{p}} f^{p}\left(a+\frac{k(b-a)}{n}\right) \frac{b-a}{n}-\frac{1}{B^{q}} g^{q}\left(a+\frac{k(b-a)}{n}\right) \frac{b-a}{n}\right]\right\}^{2} .
\end{aligned}
$$


Since

$$
\frac{1}{p}+\frac{1}{q}=1
$$

we have

$$
\begin{gathered}
{\left[A^{p}-\sum_{k=1}^{n} f^{p}\left(a+\frac{k(b-a)}{n}\right) \frac{b-a}{n}\right]^{\frac{1}{p}}\left[B^{q}-\sum_{k=1}^{n} g^{q}\left(a+\frac{k(b-a)}{n}\right) \frac{b-a}{n}\right]^{\frac{1}{q}}} \\
\geq A B-\sum_{k=1}^{n} f^{p}\left(a+\frac{k(b-a)}{n}\right) g^{q}\left(a+\frac{k(b-a)}{n}\right)\left(\frac{b-a}{n}\right) \\
\quad-\frac{A B}{q}\left\{\sum_{k=1}^{n}\left[\frac{1}{A^{p}} f^{p}\left(a+\frac{k(b-a)}{n}\right)-\frac{1}{B^{q}} g^{q}\left(a+\frac{k(b-a)}{n}\right)\right]\left(\frac{b-a}{n}\right)\right\}^{2} .
\end{gathered}
$$

In view of the hypotheses that $f(x), g(x)$ are positive Riemann integrable functions on $[a, b]$, we conclude that $f(x) g(x), f^{p}(x)$ and $g^{q}(x)$ are also integrable on $[a, b]$. Passing the limit as $n \rightarrow \infty$ in both sides of inequality (22), we obtain inequality (20). The proof of Theorem 3.1 is completed.

\section{Competing interests}

The author declares that he has have no competing interests.

\section{Acknowledgements}

The author would like to express his sincere thanks to the anonymous referees for making great efforts to improve this paper. This work was supported by the NNSF of China (Grant No. 61073121), and the Fundamental Research Funds for the Central Universities (No. 13ZD19).

Received: 27 June 2013 Accepted: 3 October 2013 Published: 08 Nov 2013

\section{References}

1. Aczél, J: Some general methods in the theory of functional equations in one variable. New applications of functional equations. Usp. Mat. Nauk 11(3), 3-68 (1956) (in Russian)

2. Popoviciu, T: On an inequality. Gaz. Mat. Fiz., Ser. A 11(64), 451-461 (1959) (in Romanian)

3. Vasić, PM, Pečarić, JE: On Hölder and some related inequalities. Mathematica 25, 95-103 (1982)

4. Farid, G, Pečarić, J, Ur Rehman, A: On refinements of Aczél's, Popoviciu, Bellman's inequalities and related results. J. Inequal. Appl. 2010, 579567 (2010)

5. Tian, J: Reversed version of a generalized sharp Hölder's inequality and its applications. Inform. Sci. 201, 61-69 (2012)

6. Tian, J, Hu, XM: A new reversed version of a generalized sharp Hölder's inequality and its applications. Abstr. Appl. Anal. 2013, Article ID 901824 (2013)

7. Tian, J, Hu, XM: Refinements of generalized Hölder's inequality. J. Math. Inequal. 7(4), 701-710 (2013)

8. Tian, JF: Property of a Hölder-type inequality and its application. Math. Inequal. Appl. 16(3), 831-841 (2013)

9. Tian, JF, Wang, S: Refinements of generalized Aczél's inequality and Bellman's inequality and their applications. J. Appl. Math. 2013, Article ID 645263 (2013)

10. Wu, S: Improvement of Aczél's inequality and Popoviciu's inequality. J. Inequal. Appl. 2007, Article ID 72173 (2007) doi:10.1155/2007/72173

11. Zhou, X: Some generalizations of Aczél, Bellman's inequalities and related power sums. J. Inequal. Appl. 2012, 130 (2012)

12. Wu, S: A further generalization of Aczél's inequality and Popoviciu's inequality. Math. Inequal. Appl. 10(3), 565-573 (2007)

13. Beckenbach, EF, Bellman, R: Inequalities. Springer, Berlin (1983)

14. Tian, JF: Reversed version of a generalized Aczél's inequality and its application. J. Inequal. Appl. 2012, 202 (2012)

10.1186/1029-242X-2013-497

Cite this article as: Tian: A sharpened and generalized version of Aczél-Vasić-Pečarić inequality and its application. Journal of Inequalities and Applications 2013, 2013:497 